\title{
Refractory infantile high-grade glioma containing TRK-fusion responds to larotrectinib
}

\author{
Torin Waters ${ }^{1}$, Steven Moore ${ }^{1}$, Yutaka Sato ${ }^{1}$, Brian Dlouhy ${ }^{1}$, and Mariko Sato ${ }^{2}$ \\ ${ }^{1}$ University of Iowa \\ ${ }^{2}$ University of Iowa Children's Hospital
}

September 11, 2020

\begin{abstract}
High-grade gliomas (HGGs) are a challenging group of brain tumors. Identification of targetable genetic alterations such as TRK fusions are providing new treatment options. We discuss an infant who presented with hydrocephalus and a cystic mass at birth, while a solid tumor component of the mass was only appreciated later. Tumor analysis was consistent with HGG containing a TRK fusion. Despite treatment with conventional chemotherapy, his tumor progressed in size. He was started on larotrectinib and experienced a significant decrease in tumor burden. This case demonstrates that targeted therapy may be effective for a subset of TRK fusion-positive CNS tumors.
\end{abstract}

\section{Case Report:}

Our patient is a 17-month old male who was found to have hydrocephalus on his 32-week fetal ultrasound. An MRI at birth (34 weeks, 4 days gestational age) revealed a large right-sided cystic mass and intraventricular hemorrhage, thought to be consistent with congenital hydrocephalus (Fig. 1A). A ventriculoperitoneal shunt was placed at one month of age due to increasing ventricular size and signs of increased intracranial pressure, including macrocephaly, nystagmus, and significant visual impairment. At seven months of age, a repeat MRI with intravenous contrast material was obtained, which showed a large cystic mass with a peripheral, avidly enhancing solid component (Fig. 1B). The concern for a neoplastic process increased, prompting surgical intervention. A frontotemporoparietal craniotomy and near-complete tumor resection were performed without complication (Fig. 1C). The child had left-sided extremity and truncal weakness, though these symptoms are improving, and he is now crawling and pulling to stand.

The pathology of the patient's tumor was complex. While a portion of the resected tissue had features of desmoplastic infantile ganglioglioma, a WHO grade I tumor (Fig. 2A-D), other features were more suggestive of a high-grade neuroepithelial tumor, or HGG (Fig. 2E-Q). See Figure 2 text for additional details. Notably, transcriptome sequencing of tumor RNA identified an EML4-NTRK3 fusion.

At 8 months of age, following surgical resection, the patient was started on chemotherapy with vincristine, cisplatin, etoposide, and cyclophosphamide. He completed four cycles of chemotherapy three months later.

Repeat imaging two months post-therapy failed to show reduction of the tumor volume (Fig. 1D). Given the patient's young age, radiation therapy was deferred. He was started on larotrectinib, a TRK inhibitor, for salvage therapy. His repeat MRI (Fig. 1E) two months later revealed a substantial decrease in size of his residual tumor. He continues on larotrectinib $100 \mathrm{mg} / \mathrm{m}^{2} /$ dose twice daily and has shown no signs of progression to date, after nine months on therapy.

\section{Discussion :}


Infantile high-grade glioma, while having superior outcomes compared to gliomas in older pediatric patients, has a $50 \%$ chance of progression despite conventional chemotherapy and radiotherapy treatment ${ }^{3}$. Our case highlights a challenging tumor, which presented as hydrocephalus at birth, but was not diagnosed as a neoplastic process until 7 months of age. The tumor was also initially identified as desmoplastic infantile ganglioglioma, and while it did have features of this diagnosis, aggressive features consistent with high-grade glioma were more prevalent. The finding of anEML4-NTRK3 fusion oncogene provided an indication for a trial of larotrectinib, after progression on conventional chemotherapy. His significant response to larotrectinib provides further evidence that TRK inhibitors can be effective in TRK fusion-positive CNS tumors.

Based on phase I/II studies to date, in both pediatric and adult patients, the side effect profile of larotrectinib is tolerable. In a recent review of three phase I/II trials, dose reduction of larotrectinib due to side effects only occurred in $8 \%$ of patients with TRK-fusion positive tumors, and discontinuation due to a drugrelated adverse event only occurred in $2 \%$ of the overall cohort ${ }^{6}$. The most common adverse events were ALT elevation in $3 \%$ of patients, anemia in $2 \%$, and neutropenia in $2 \%$. Compared with the numerous adverse effects of conventional chemotherapy, including bone marrow suppression, infertility, and secondary malignancies, among others, the acute effects of larotrectinib are tolerable. While there is limited side effect data for larotrectinib in infants, the devastating neurocognitive effects of radiation therapy in this age group made a trial of targeted therapy an appealing alternative. We are monitoring carefully for side effects of TRK inhibitor therapy in our patient, but he has done well to date.

Larotrectinib has shown promising results in patients with TRK-fusion positive tumors, particularly infantile fibrosarcoma, in which larotrectinib use for progressive tumors has prevented debilitating surgeries in a number of patients ${ }^{5}$. Due to emerging evidence of the promising effects of TRK fusion-targeted therapy, numerous clinical trials are ongoing in both adults and children with relapsed cancer.

With regards to infantile high-grade glioma in particular, Ziegler et al. ${ }^{7}$ also described an infant with an encouraging response to larotrectinib, following disease progression with a standard chemotherapy regimen. That patient's tumor contained anETV6-NTRK3 fusion, as opposed to the EML4-NTRK3 fusion found in our patient's tumor. Our patient's tumor also contained pathologic features of desmoplastic infantile ganglioglioma, a rare, grade I pediatric brain tumor that can sometimes develop malignant potential.

Our case provides a promising example of larotrectinib's utility in infants with TRK fusion-positive HGGs. Clinical trials, such as the Children's Oncology Group study APEC1621A, are underway to investigate the use of larotrectinib in a variety of TRK fusion-positive solid and CNS tumors. This study is only enrolling patients over twelve months of age, however, and our report helps demonstrate the potential benefit for infants with refractory HGG who would otherwise have a poor prognosis. While questions regarding duration of therapy and long-term side effects of larotrectinib remain, we would advocate for the establishment of a clinical trial including infants with TRK fusion-positive HGGs.

\section{Conflict of Interest:}

The authors declare that there is no conflict of interest.

\section{References:}

1. El-Ayadi M, Ansari M, Sturm D, et al. High-grade glioma in very young children: a rare and particular patient population. Oncotarget . 2017;8(38):64564-64578

2. Gielen GH, Gessi M, Buttarelli FR, et al. Genetic Analysis of Diffuse High-Grade Astrocytomas in Infancy Defines a Novel Molecular Entity. Brain Pathol . 2015;25(4):409-417

3. Guerreiro Stucklin AS, Ryall S, Fukuoka K, et al. Alterations in ALK/ROS1/NTRK/MET drive a group of infantile hemispheric gliomas. Nat Commun . 2019;10(1):4343

4. Wu G, Diaz AK, Paugh BS, et al. The genomic landscape of diffuse intrinsic pontine glioma and pediatric non-brainstem high-grade glioma. Nat Genet . 2014;46(5):444-450

5. Laetsch TW, DuBois SG, Mascarenhas L, et al. Larotrectinib for paediatric solid tumours harbouring NTRK gene fusions: phase 1 results from a multicentre, open-label, phase $1 / 2$ study [published 
correction appears in Lancet Oncol. 2018 May;19(5):e229]. Lancet Oncol . 2018;19(5):705-714

6. Hong DS, DuBois SG, Kummar S, et al. Larotrectinib in patients with TRK fusion-positive solid tumours: a pooled analysis of three phase 1/2 clinical trials. Lancet Oncol . 2020;21(4):531-540

7. Ziegler DS, Wong M, Mayoh C, et al. Brief Report: Potent clinical and radiological response to larotrectinib in TRK fusion-driven high-grade glioma. Br J Cancer . 2018;119(6):693-696

Figure Legends:

Figure 1. Brain imaging. A) T1-weighted brain MRI without intravenous contrast at 1-day of age showed a large extra-axial cystic mass in the right convexity and temporal lobe signal alteration. Also present was intraventricular hemorrhage with fluid-fluid levels in the dilated lateral ventricles. B)-E) T1weighted brain MRI images with intravenous contrast. B) At 7-months of age, the previously noted cystsic lesion demonstrated an extensive and avidly enhancing solid component at the periphery. C) At 8-months of age, following surgical resection, there is residual enhancing mass in the posterior aspect of the tumor bed. D) At 13-months of age, 2 months after completion of conventional chemotherapy, there is progressive residual mass. E) At 15-months of age, 2 months after starting larotrectinib, rapid reduction of the residual tumor is seen.

Figure 2. Tumor histopathology. The desmoplastic portion of the tumor is comprised of spindle cells (upper portion of panel A) with abundant reticulin (B). Spindle cells are polyphenotypic. The expression of S100 protein (C) and smooth muscle-specific actin (SMSA; D) are shown here. Arrows in A-D mark the glia limitans border between abnormal cerebral cortex and desmoplastic neoplasm. Away from the desmoplastic components, the cerebral cortex contains nodular layer I clusters of neurons (E-H; arrows in E-G) and cortical dysplasia (I-K), with disorganized lower layers of the cortex (I) containing dysmorphic neurons ( $\mathrm{J}$ and $\mathrm{K}$ ). Arrows in $\mathrm{K}$ point to neuronal cell bodies positive for neurofilament. A minority of the tissue resection consisted of high-grade neuroepithelial tumor. Malignant neuroepithelial neoplasm (L-O) is hypercellular with foci of necrosis (M), vascular proliferation (arrows in N and O), diffuse S100 protein expression (O), and high Ki67 proliferative index $(\mathrm{P})$. There was no immunoreactivity for the mutant proteins BRAF V600E or H3K27M. Clusters of malignant cells were present in postoperative drain fluid, presumably CSF (Q). 
Figure 1
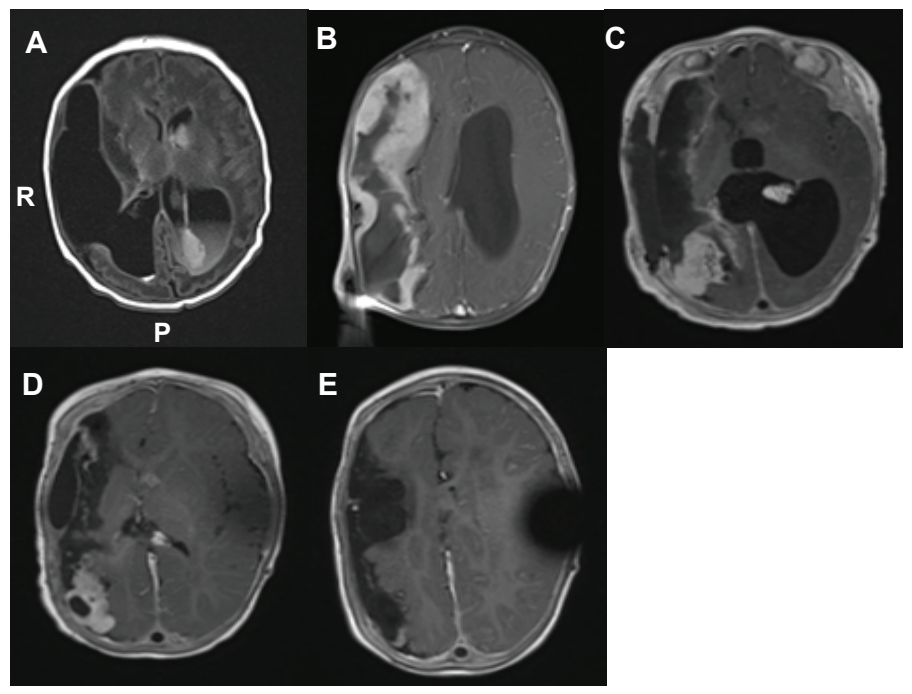
Figure 2
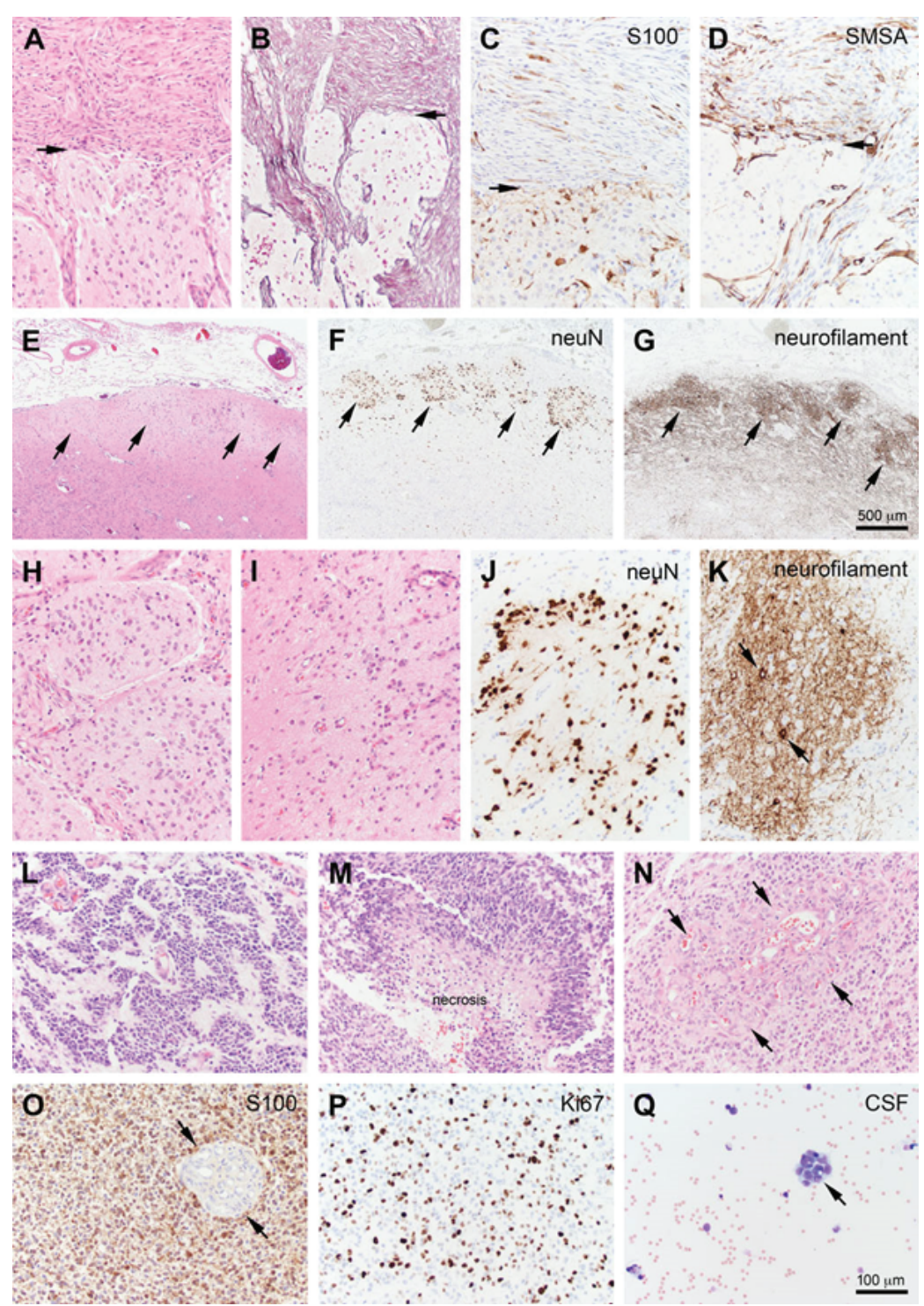